\title{
Brane in 6D with an increasing gravitational trapping potential
}

\author{
Merab Gogberashvili* \\ Andronikashvili Institute of Physics, 6 Tamarashvili Str., Tbilisi 380077, Georgia \\ Douglas Singleton ${ }^{\dagger}$ \\ Physics Department, CSU Fresno, 2345 East San Ramon Avenue, M/S 37 Fresno, California 93740-8031, USA
}

(Received 27 May 2003; published 15 January 2004)

\begin{abstract}
A new solution to the Einstein equations in $1+5$ spacetime with an embedded $1+3$ brane is given. This solution localizes the zero modes of all kinds of matter fields and four-gravity on the $(1+3)$ brane by an increasing, transverse gravitational potential. This localization occurs despite the fact that the gravitational potential is not a decreasing exponential, and asymptotically approaches a finite value rather than zero.
\end{abstract}

DOI: 10.1103/PhysRevD.69.026004

PACS number(s): 11.25.Wx, 04.50.+h, 11.10.Kk, 98.80.Cq

The main question of brane models is how to localize fields on the brane. To localize multidimensional fields on the brane the effective "coupling" constants appearing after integration of the Lagrangian over the extra coordinates must be nonvanishing and finite. For reasons of economy one would like to have a single, universal trapping mechanism that works for all fields. It is natural to try gravitational trapping of the physical fields on the brane, since gravity is known to have a universal coupling with all matter fields.

In $(1+4)$-dimensional models the following results were established: spin 0 and spin 2 fields are localized on the brane with a decreasing, exponential gravitational warp factor, spin 1/2 fields are localized on the brane with an increasing warp factor $[1,2]$, and spin 1 fields are not localized at all [3]. For the case of $1+5$ dimensions it was found that spin 0,1 and 2 fields are localized on the brane with a decreasing warp factor and spin $1 / 2$ fields are localized on the brane with an increasing warp factor [5]. So in both $(1+4)$ - and $(1+5)$-space models one is required to introduce some nongravitational interaction in order to localize the standard model particles.

Here we want to show that zero modes of spin $0,1 / 2,1$, and 2 fields can all be localized on the brane in a $1+5$ space by an increasing warp factor which is not an exponential. A similar solution for a $(2+4)$-signature metric was found in previous work [6]. Having a growing gravitational potential (warp factor) is opposite to the choice of the RandallSundrum model where the warp factor maximum is on the brane [1]. However, Newton's law still holds on the brane as a result of the cancellation mechanism introduced in [4] which allows both increasing and decreasing types of gravitational potential.

The action of the gravitating system in six dimensions can be written in the form

$$
S=\int d^{6} x \sqrt{-{ }^{6} g}\left[\frac{M^{4}}{2}\left({ }^{6} R+2 \Lambda\right)+L\right],
$$

where $\sqrt{-{ }^{6} g}$ is the determinant, $M$ is the fundamental scale,

\footnotetext{
*Electronic address: gogber@hotmail.com

†Electronic address: dougs@csufresno.edu
}

${ }^{6} R$ is the scalar curvature, $\Lambda$ is the cosmological constant, and $L$ is the Lagrangian of matter fields. All of these quantities are six dimensional.

Einstein's six-dimensional equations with stress-energy tensor $T_{A B}$ are

$$
R_{A B}-\frac{1}{2} g_{A B}{ }^{6} R=\frac{1}{M^{4}}\left(\Lambda g_{A B}+T_{A B}\right) .
$$

Capital latin indices run over $A, B, \ldots=0,1,2,3,5,6$.

We will look for solutions of Eq. 2 that contain fourdimensional Minkowski geometry with the following Ansatz:

$$
d s^{2}=\phi^{2}\left(x^{i}\right) \eta_{\alpha \beta}\left(x^{\nu}\right) d x^{\alpha} d x^{\beta}+g_{i j}\left(x^{i}\right) d x^{i} d x^{j} .
$$

The metric of ordinary four-dimensional space, $\eta_{\alpha \beta}\left(x^{\nu}\right)$, has the signature $(+,-,-,-)$. The greek indices $\alpha, \beta, \ldots$ $=0,1,2,3$ refer to the coordinates of these four-dimensions, while small latin indices $i, j, \ldots=5,6$ refer to coordinates of the transverse space. It is assumed that the Ansatz (3) depends only on the extra coordinates $x^{i}$ through the fourdimensional conformal factor $\phi^{2}$ and the metric tensor of transversal two-space, $g_{i j}$.

Since gravity in the transverse two-space is trivial, the metric of the extra space, $g_{i j}\left(x^{i}\right)$, has only one independent component. We will choose its diagonal component as independent:

$$
g_{i j}\left(x^{i}\right)=-\delta_{i j} \lambda(r),
$$

where $\delta_{i j}$ is the metric of Euclidean two-space.

We require cylindrical symmetry of the transverse twospace. It is convenient to write the two extra spatial dimensions $x^{5}$ and $x^{6}$ using polar coordinates $(r, \theta)$, where $0 \leqslant r$ $=\sqrt{x_{5}^{2}+x_{6}^{2}}<\infty$ and $0 \leqslant \theta<2 \pi$. Thus the Ansatz (3) takes the form

$$
d s^{2}=\phi^{2}(r) \eta_{\alpha \beta}\left(x^{\nu}\right) d x^{\alpha} d x^{\beta}-\lambda(r)\left(d r^{2}+r^{2} d \theta^{2}\right) .
$$

This metric is slightly different from the metrics investigated in other $(1+5)$-space brane models $[5,7,8]$. The independent metric function of the extra space, $\lambda(r)$, serves as a conformal factor for the Euclidean two-dimensional metric 
of the transverse space just as the function $\phi^{2}(r)$ does for the four-dimensional part. Usually in six-dimensional Ansätze the independent metric function multiples only the angular part $\left(d \theta^{2}\right)$ of the metric (5) and corresponds to a cone like geometry of a string like defect with a singularity at the center $r=0$. We want to look for nonsingular solutions, and so we choose the metric to take the form (5) where the extra part is conformally equivalent to Euclidean two-space.

The nonzero components of the stress-energy tensor $T_{A B}$ are assumed to be

$$
T_{\mu \nu}=-g_{\mu \nu} F(r), \quad T_{i j}=-g_{i j} K(r), \quad T_{i \mu}=0 .
$$

The two source functions $F(r)$ and $K(r)$ are assumed to depend only on the radial coordinate $r$.

For the four-dimensional Einstein equations outside the brane we require the ordinary form without a cosmological term,

$$
R_{\mu \nu}-\frac{1}{2} \eta_{\mu \nu} R=0
$$

The Ricci tensor in four dimensions $R_{\alpha \beta}$ is constructed from the four-dimensional metric tensor $\eta_{\alpha \beta}\left(x^{\nu}\right)$ in the standard way. Then with the Ansätze (5) and (6) the Einstein field equations (2) become

$$
\begin{aligned}
& 3 \frac{\phi^{\prime \prime}}{\phi}+3 \frac{\phi^{\prime}}{r \phi}+3 \frac{\left(\phi^{\prime}\right)^{2}}{\phi^{2}}+\frac{1}{2} \frac{\lambda^{\prime \prime}}{\lambda}-\frac{1}{2} \frac{\left(\lambda^{\prime}\right)^{2}}{\lambda^{2}}+\frac{1}{2} \frac{\lambda^{\prime}}{r \lambda} \\
&=\frac{\lambda}{M^{4}}(F(r)-\Lambda), \\
& \frac{\phi^{\prime} \lambda^{\prime}}{\phi \lambda}+2 \frac{\phi^{\prime}}{r \phi}+3 \frac{\left(\phi^{\prime}\right)^{2}}{\phi^{2}}=\frac{\lambda}{2 M^{4}}(K(r)-\Lambda), \\
& 2 \frac{\phi^{\prime \prime}}{\phi}-\frac{\phi^{\prime} \lambda^{\prime}}{\phi \lambda}+3 \frac{\left(\phi^{\prime}\right)^{2}}{\phi^{2}}=\frac{\lambda}{2 M^{4}}(K(r)-\Lambda),
\end{aligned}
$$

where the prime indicates $\partial / \partial r$. These equations are for the $\alpha \alpha, r r$, and $\theta \theta$ components, respectively.

Subtracting the $r r$ from the $\theta \theta$ equation and multiplying by $\phi / \phi^{\prime}$ we arrive at

$$
\frac{\phi^{\prime \prime}}{\phi^{\prime}}-\frac{\lambda^{\prime}}{\lambda}-\frac{1}{r}=0
$$

This equation has the solution

$$
\lambda(r)=\frac{\rho^{2} \phi^{\prime}}{r},
$$

where $\rho$ is an integration constant with units of length.

We want to find a solution $\phi(r)$ [and thereby also $\lambda(r)$ via Eq. (10)] which will provide a universal, gravitational trapping for all kinds of matter fields. In the above the brane is located at the origin $r=0$. From Eq. (10) we see that to avoid singularities the function $\lambda \propto \phi^{\prime}$ cannot change sign and should approach zero only on the brane $r \rightarrow 0$ and at infinity $r \rightarrow \infty$. Therefore we must look for a solution $\phi$ which is a monotone function of $r$ and increases or decreases from the brane to some finite value at infinity.

A field will be trapped on the brane if the integral over the extra coordinates $r, \theta$ converges. From the metric Ansatz (5) and the solution for $\lambda(r)$ in Eq. (10) the general expression for these integrals is

$$
\begin{gathered}
\int_{0}^{2 \pi} d \theta \int_{0}^{\infty} d r \phi^{c}\left(\phi^{\prime}\right)^{d} \sqrt{-{ }^{6} g} \\
=2 \pi \int_{0}^{\infty} d r \phi^{c}\left(\phi^{\prime}\right)^{d} r \phi^{4} \lambda \sqrt{-\eta} \\
=2 \pi \rho^{2} \int_{0}^{\infty} d r \phi^{n}\left(\phi^{\prime}\right)^{m} \sqrt{-\eta},
\end{gathered}
$$

where $\eta$ is the determinant of the ordinary four-dimensional metric tensor, $c$ and $d$ are numbers that depend on the type of field being trapped, and $n=c+4$ and $m=d+1$. To have localized physical fields on the brane the integral of $\phi^{n}\left(\phi^{\prime}\right)^{m}$ must go to zero fast enough as $r \rightarrow \infty$, and not have any essentially singularities in the range $0 \leqslant r \leqslant \infty$. For the fields that we will consider (spin 0 , spin $1 / 2$, spin 1 , and spin 2), $m$ is positive while $n$ can be either positive or negative. One standard possibility is to have an exponential warp factor, $\phi(r) \propto e^{-c r}[5,7,8]$. In this case for fields with $n>0 \phi^{n}$ and $\left(\phi^{\prime}\right)^{m}$ go to zero as $r \rightarrow \infty$ and the integral (11) converges and the fields will be trapped. However, for $n<0$ (for example, with spinor fields) the integral diverges and the fields are not trapped.

In this work we present a different type of solution where the gravitational potential $\phi$ is not an exponential and goes to a finite value at $r=\infty$ [i.e., $\phi(\infty)=$ const], yet nevertheless is able to trap the zero modes of all types of fields since $\phi^{\prime} \rightarrow 0$. A similar nonexponential solution for the signature $2+4$ was found in previous work [6]. This type of solution is impossible in the five-dimensional case where only an exponential warp factor is possible.

Based on the above discussion we choose the boundary conditions on the brane as

$$
\phi(r \leqslant \epsilon) \approx 1, \quad \phi^{\prime}(r \leqslant \epsilon) \approx 0,
$$

where $\epsilon$ is the brane width. The boundary conditions at infinity are

$$
\phi(r \rightarrow \infty) \rightarrow a, \quad \phi^{\prime}(r \rightarrow \infty) \rightarrow 0,
$$

where $a>1$ is some constant. Since the function $\phi^{\prime}$ is proportional to the metric of the extra two-space, $g_{i j}$, the boundary conditions (12) and (13) imply that on the brane and at infinity the effective geometry of space-time is fourdimensional.

The energy-momentum conservation equation

$$
\nabla^{A} T_{A B}=\frac{1}{\sqrt{-{ }^{6} g}} \partial_{A}\left(\sqrt{-{ }^{6} g} T^{A B}\right)+\Gamma_{C D}^{B} T^{C D}=0
$$


gives a relationship between the two source functions $F(r)$ and $K(r)$ in Eq. 6. In terms of the Ansatz functions the conservation law reads

$$
K^{\prime}+4 \frac{\phi^{\prime}}{\phi}(K-F)=0 \text {. }
$$

This equation places a restriction on the form of the source functions, which will allow us to determine a simple form for $F(r)$ and $K(r)$. We assume that they are smooth functions of the radial coordinate $r$ and describe a continuous matter distribution within a core of radius $\epsilon$. We also assume that they decrease rapidly when $r>\epsilon$. Below we will find that the transverse gravitational potential $\phi^{2}(r)$ can be a growing function as one moves off the brane, so the factor $1 / \phi^{2}(r)$ has $\delta$-like behavior. Thus outside the core we will set the source functions proportional to $1 / \phi^{2}$. This form satisfies Eq. (15) with the sources functions given by

$$
F(r>\epsilon)=\frac{f}{2 \phi^{2}}, \quad K(r>\epsilon)=\frac{f}{\phi^{2}},
$$

where $f$ is some constant.

Now we shall find the outer solution $(r>\epsilon)$ of the sixdimensional Einstein equations (2) for the brane when the metric and matter energy-momentum tensor have the general forms given in Eqs. (5) and (16), respectively. The system (8), after the insertion of Eqs. (10) and (16), has only one independent equation. Taking either the $r r$ or $\theta \theta$ component of these equations and multiplying by $r \phi^{4}$ gives

$$
r \phi^{3} \phi^{\prime \prime}+\phi^{3} \phi^{\prime}+3 r \phi^{2}\left(\phi^{\prime}\right)^{2}=\frac{\rho^{2} \phi^{4} \phi^{\prime}}{2 M^{4}}\left(\frac{f}{\phi^{2}}-\Lambda\right) .
$$

Taking the first integral of this equation and setting the integration constant to zero yields [6]

$$
r \phi^{\prime}=A\left(-\phi^{2}+a^{2}\right)
$$

where we have introduced the parameters

$$
A=\frac{\rho^{2} \Lambda}{10 M^{4}}, \quad a^{2}=\frac{5 f}{3 \Lambda} .
$$

From the Eq. (18) we see that to have an increasing function $\phi\left(\phi^{\prime}>0\right)$ we must require the following conditions:

$$
a>\phi, \quad \Lambda>0, \quad f>0 .
$$

Equation (18) is easy to integrate:

$$
\phi=a \tanh \left[b \ln \left(\frac{r}{c}\right)\right]=a \frac{r^{2 b}-c^{2 b}}{r^{2 b}+c^{2 b}}
$$

where $b=A a$. The integration constant $c$ can be fixed from the boundary conditions (12),

$$
c^{2 b}=\epsilon^{2 b} \frac{a-1}{a+1},
$$

where $\epsilon$ is the brane width.

It is easy to show that four-dimensional gravity is localized on the brane by the solution (21) in spite of its growing character. We will consider only spin-2 modes, which are transverse, traceless fluctuations $H_{\mu \nu}$ around the background metric (5):

$$
d s^{2}=\phi^{2}(r)\left(\eta_{\mu \nu}+H_{\mu \nu}\right) d x^{\mu} d x^{\nu}-\frac{\rho^{2} \phi^{\prime}}{r}\left(d r^{2}+r^{2} d \theta^{2}\right),
$$

where $\phi$ has the form (21).

In the gauge $\nabla^{\mu} H_{\mu \nu}=H=0$, Einstein's equations reduce to the form of the linearized equations

$$
\frac{1}{\sqrt{-g}} \partial_{A}\left(\sqrt{-g} g^{A B} \partial_{B} H_{\mu \nu}\right)-2 \Lambda H_{\mu \nu}=0 .
$$

We look for solutions of the form

$$
H_{\mu \nu}\left(x^{A}\right)=h_{\mu \nu}\left(x^{\mu}\right) \sum_{l m} \sigma_{m}(r) e^{i l \theta},
$$

where $h_{\mu \nu}\left(x^{\mu}\right)$ satisfy the four-dimensional field equations

$$
\left(\Delta-2 \Lambda \phi^{2}\right) h_{\mu \nu}\left(x^{\mu}\right)=-m_{0}^{2} h_{\mu \nu}\left(x^{\mu}\right),
$$

with the definition of

$$
\Delta=\frac{1}{\sqrt{-\eta}} \partial_{\mu}\left(\sqrt{-\eta} \quad \eta^{\mu \nu} \partial_{\nu}\right)
$$

Note that the presence of the cosmological constant term $2 \Lambda \phi^{2}$ in Eq. (26), which appears in most studies of the localization of spin-2 fields $[1,5,7,8]$, is problematic since it implies a negative energy, tachyonic spin- 0 mode for the fluctuation $h_{\mu \nu}$ on the brane. One could consider fluctuations coming from the extra coordinates, which might have a chance to cancel this contribution. Without a satisfactory quantum theory of gravity it is not certain how to properly resolve this difficulty, and we will not consider its resolution here.

Using Eq. (26), Eq. (24) reduces to

$$
\sigma^{\prime \prime}+\left(\frac{1}{r}+4 \frac{\phi^{\prime}}{\phi}\right) \quad \sigma^{\prime}+\left(\frac{m_{0}^{2} \rho^{2}}{r} \frac{\phi^{\prime}}{\phi^{2}}-\frac{l^{2}}{r^{2}}\right) \quad \sigma=0 .
$$

It is easy to see that this equation has the zero-mass $\left(m_{0}\right.$ $=0)$ and $s$-wave $(l=0)$ constant solution $\sigma_{0}=$ const. Substitution of this zero mode into the Einstein-Hilbert action leads to

$$
S^{(0)} \sim \sigma_{0}^{2} \int_{0}^{2 \pi} d \theta \int_{0}^{\infty} d r \frac{\sqrt{-^{6} g}}{\phi^{2}} \int d^{4} x\left[\partial^{\alpha} h^{\mu \nu} \partial_{\alpha} h_{\mu \nu}+\cdots\right] .
$$


To have the four-dimensional spin-2 graviton localized on the brane requires that the integral over $r$ and $\theta$ (which corresponds to the four-dimensional Planck scale) converge

$$
\begin{aligned}
m_{P l}^{2} & =2 \pi M^{4} \int_{0}^{\infty} d r \frac{r \phi^{4} \lambda}{\phi^{2}} \\
& =2 \pi \rho^{2} M^{4} \int_{0}^{\infty} \phi^{2} \phi^{\prime} d r=2 \pi \rho^{2} M^{4} \int_{1}^{a} \phi^{2} d \phi \\
& =\frac{2}{3} \pi \rho^{2} M^{4}\left(a^{3}-1\right) .
\end{aligned}
$$

Now we want to check that zero modes of matter fields are also localized on the brane with the nonexponential, increasing warp factor (21).

For spin-0 fields in six dimensions we assume that the fields are independent of the extra coordinates so that the action can be cast in the form

$$
\begin{aligned}
S_{\Phi} & =-\frac{1}{2} \int d^{6} x \sqrt{-{ }^{6} g} g^{A B} \partial_{A} \Phi \partial_{B} \Phi \\
& =-\pi \rho^{2} \int_{1}^{a} \phi^{2} d \phi \int d^{4} x \sqrt{-\eta} \eta^{\mu \nu} \partial_{\mu} \Phi \partial_{\nu} \Phi .
\end{aligned}
$$

The integral over the extra coordinates in Eq. (31) is the same as for the spin-2 case above. Thus the integral is finite and the spin-0 field is localized on the brane.

The action for a $U(1)$ vector gauge field in the case of constant extra components $\left(A_{i}=\right.$ const $)$ reduces to the fourdimensional Maxwell action multiplied by an integral over the extra coordinates

$$
\begin{aligned}
S_{A}= & -\frac{1}{4} \int d^{6} x \sqrt{-{ }^{6} g} g^{A B} g^{M N} F_{A M} F_{B N} \\
& =-\frac{\pi}{2} \rho^{2} \int_{1}^{a} d \phi \int d^{4} x \sqrt{-\eta} \eta^{\mu \nu} \eta^{\alpha \beta} F_{\mu \alpha} F_{\nu \beta} .
\end{aligned}
$$

This integral is also finite, and the gauge field is localized on the brane.

In the case of spinor fields we introduce the sechsbein $h_{M}^{\bar{M}}$, where $\bar{M}, \bar{N}, \ldots$ denotes local Lorentz indices. The spin connection is defined as

$$
\begin{aligned}
\omega_{M}^{\bar{M} \bar{N}}= & \frac{1}{2} h^{N \bar{M}}\left(\partial_{M} h_{N}^{\bar{N}}-\partial_{N} h_{M}^{\bar{N}}\right)-\frac{1}{2} h^{N \bar{N}}\left(\partial_{M} h_{N}^{\bar{M}}-\partial_{N} h_{M}^{\bar{M}}\right) \\
& -\frac{1}{2} h^{P \bar{M}} h^{Q \bar{N}}\left(\partial_{P} h_{Q \bar{R}}-\partial_{Q} h_{P \bar{R}}\right) h_{M}^{\bar{R}} .
\end{aligned}
$$

The nonvanishing components of the spin connection for the background metric Ansatz are

$$
\omega_{\mu}^{\bar{r} \bar{\nu}}=\delta_{\mu}^{\bar{\nu}} \frac{\sqrt{r \phi^{\prime}}}{\rho}, \quad \omega_{\theta}^{\bar{r} \bar{\theta}}=\sqrt{\frac{r}{\phi^{\prime}}} \partial_{r}\left(\sqrt{r \phi^{\prime}}\right) .
$$

Therefore, the covariant derivatives have the form

$$
\begin{gathered}
D_{\mu} \Psi=\left(\partial_{\mu}+\frac{1}{2} \omega_{\mu}^{\bar{r} \nu} \gamma_{r} \gamma_{\nu}\right) \Psi, \quad D_{r} \Psi=\partial_{r} \Psi, \\
D_{\theta} \Psi=\left(\partial_{\theta}+\frac{1}{2} \omega_{\theta}^{\bar{r} \bar{\theta}} \gamma_{r} \gamma_{\theta}\right) \Psi,
\end{gathered}
$$

where $\gamma_{\nu}, \gamma_{r}, \gamma_{\theta}$ are gamma matrices.

We are looking for solutions of the form $\Psi\left(x^{A}\right)$ $=\psi\left(x^{\nu}\right) B(r)$, where $\psi$ satisfies the massless fourdimensional Dirac equation, $\gamma^{\nu} \partial_{\nu} \psi=0$. Then the sixdimensional massless Dirac equation

$$
\Gamma^{\mu} D_{\mu} \Psi+\Gamma^{r} D_{r} \Psi+\Gamma^{\theta} D_{\theta} \Psi=0,
$$

where $\Gamma^{A}=h_{\bar{B}}^{A} \gamma^{B}$ are the six-dimensional curved space gamma matrices, reduces to

$$
\left[\partial_{r}+2 \frac{\phi^{\prime}}{\phi}+\frac{1}{2} \frac{1}{\sqrt{r \phi^{\prime}}} \partial_{r}\left(\sqrt{r \phi^{\prime}}\right)\right] B(r)=0 .
$$

The solution of this equation with the integration constant, taken as 1 is

$$
B(r)=\phi^{-2}\left(r \phi^{\prime}\right)^{-1 / 4} .
$$

Using the solution from Eq. (21) one finds that $B(r) \rightarrow \infty$ as $r \rightarrow \infty$. This happens since $r \phi^{\prime} \rightarrow 0$ as $r \rightarrow \infty$. This appears to imply that the total spinor wave function, $\Psi\left(x^{A}\right)$, does not have good asymptotic behavior. However, the effective wave function is $\Psi\left(x^{A}\right)\left(-{ }^{6} g\right)^{1 / 4} \propto B(r) \sqrt{\rho^{2} \phi^{4} \phi^{\prime}} \rightarrow \rho\left(\phi^{\prime} / r\right)^{1 / 4}$, which goes to zero as $r \rightarrow \infty$. The action of the spin-1/2 field takes the form

$$
\begin{aligned}
S_{\Psi} & =\int d^{6} x \sqrt{-{ }^{6} g} \bar{\Psi} i \Gamma^{A} D_{A} \Psi \\
& =2 \pi \rho^{2} \int_{0}^{\infty} d r r^{-1 / 2} \phi^{-1}\left(\phi^{\prime}\right)^{1 / 2} \int d^{4} x \sqrt{-\eta} \bar{\psi} i \gamma^{\nu} \partial_{\nu} \psi .
\end{aligned}
$$

Using the explicit form of the solution (21), one finds that the integral over $r$ in Eq. (39) is of the form

$$
\int d r \quad \sqrt{\frac{\phi^{\prime}}{r \phi^{2}}}=\frac{1}{\sqrt{a b}} \ln \left(\frac{r^{b}-c^{b}}{r^{b}+c^{b}}\right) .
$$

This integral converges if one evaluates it from $r=\epsilon$ to $r$ $=\infty$. Thus the massless Dirac fermions are also localized on the brane.

When we consider the interaction of scalars or fermions with the electromagnetic field we must make the usual replacements

$$
\partial_{i} \rightarrow \partial_{i}-i A_{i}, \quad \Psi \rightarrow e^{i A_{i} x^{i}} \Psi
$$

in the above formula for localization. Here $x^{i}$ are coordinates of transverse two-space and $A_{i}$ are constant extra components of the electromagnetic field. 
To summarize, in this paper it is shown that for a realistic form of the brane stress-energy, there exists a non-singular static solution of six-dimensional Einstein equations. This solution provides gravitational trapping of the fourdimensional gravity and matter fields on the brane without extra $\delta$-like sources. In contrast to the Randall-Sundrum case, the factor responsible for this trapping is an increasing gravitational potential. Despite this and the fact that the transverse space is infinite, the integral over the extra coordinates is convergent, and the fields are localized on the brane.

\section{ACKNOWLEDGMENTS}

This work is supported by a COBASE grant.
[1] L. Randall and R. Sundrum, Phys. Rev. Lett. 83, 3370 (1999); 83, 4690 (1999).

[2] B. Bajc and G. Gabadadze, Phys. Lett. B 474, 282 (2000).

[3] A. Pomarol, Phys. Lett. B 486, 153 (2000).

[4] M. Gogberashvili, Int. J. Mod. Phys. D 11, 1639 (2002); 11, 1635 (2002).
[5] I. Oda, Phys. Rev. D 62, 126009 (2000).

[6] M. Gogberashvili and P. Midodashvili, Phys. Lett. B 515, 447 (2001); Europhys. Lett. 61, 308 (2003).

[7] R. Gregory, Phys. Rev. Lett. 84, 2564 (2000).

[8] T. Gherghetta and M. Shaposhnikov, Phys. Rev. Lett. 85, 240 (2000). 